\title{
Erratum
}

\section{Effect of Electromagnetic Treatment on Fatigue Resistance of 2011 Aluminum Alloy}

[Journal of Multiscale Modelling, Vol. 7, No. 3 (2016) 1650004 (6 pages)]

\author{
M. A. Mohin ${ }^{* \dagger}$, H. Toofany ${ }^{*, \S}$, A. Babutskyi ${ }^{*}$, A. Lewis ${ }^{*, \|}$ and Y. G. Xu ${ }^{\dagger, * *}$ \\ ${ }^{*}$ School of Engineering and Technology \\ University of Hertfordshire, AL10 9AB, UK \\ ${ }^{\dagger}$ School of Aerospace, Transport and Manufacturing \\ Cranfield University, MK43 OAL, UK \\ ${ }^{\ddagger}$ m.mohin@herts.ac.uk \\ §hasift93@yahoo.co.uk \\ 『a.babutskyi@herts.ac.uk \\ "la.lewis@herts.ac.uk \\ **yigeng.xu@cranfield.ac.uk
}

Accepted 17 November 2016

Published 26 January 2017

H. Toofany should be H. Toofanny and his email should be hasift93@hotmail.co.uk. 